\title{
Using LabView environment for the demonstration of generator dynamic behavior
}

\author{
Imanakunova Zh.S. ${ }^{1 *}$, Satarkulov K. ${ }^{1}$, Baibagysova D.Zh. ${ }^{1}$, Kadieva A.K. ${ }^{1}$ \\ ${ }^{1}$ KSTU, Bishkek, Kyrgyzstan
}

\begin{abstract}
Under consideration is a virtual device (VD) in NI - LabVIEW environment for the demonstration of a generator dynamic behavior close to borders of its stability area. The generator is operating for the unlimited power system. Capabilities of VD are demonstrated on a specific example. It was established that dynamic modes of various complexity - from stationary mode to attractors of different types that correspond to different modes of the generator under study can be observed in the system under consideration. Identification of chaotic modes of generator operation is a topical task as deterministic chaos due to its unpredictability is certainly an inadmissible phenomenon from the point of view of dynamic stability of its operation.
\end{abstract}

\section{Introduction}

A system of equations used for the analysis of dynamical stability of generators (synchronous machines) normally relates to determinate systems of nonlinear differential equations (DSNDE).

The idea that solution of the given DSNDE with the known initial conditions is predictable at any time interval turned out to be wrong, as a chaotic solution [1$5]$ was found for such systems [6, 7].

There is some range of parameters in DSNDE, solutions in such a range although turn to be limited, but are noncyclic and unpredictable, i.e. gain random nature and therefore have non-discrete, but wideband continuous range $[6,8]$.

For electric energy systems (EES) identification of chaotic modes is a topical task, as deterministic chaos due to its unpredictability - is certainly inadmissible phenomenon from the point of view of dynamical stability in EES [9]. And besides, wideband range of chaotic oscillations will be a reason for appearing:

- large number of frequencies, some of them may match own frequencies of mechanical oscillations, for instance, shaft of turbo- or hydrogenerator, may result in their destruction due to occurrence of electromechanical resonance (EMR) [10];

- higher harmonics of current and voltage [9], that facilitate the impact upon the occurrence of additional loss of electricity in power grid elements [11].

The article shows the results of study with the help of VD-designed dependency of circular frequency deviation $\omega(t)$ from rated value $\omega_{\mu}$ for a generator operating for a system with unlimited power (SUP) with the purpose of identifying its chaotic behavior. Selection of $\omega(t)$ as a subject of research is explained by the fact that reliable operation of EES is related to value $\omega(t)$.

\section{Task description}

Nonlinear equations are solved by numerical techniques with computers, and the same approach is used while analyzing power systems stability. Conclusion of synchronous machines stability is made on the basis of change in angles $\delta_{i}$ their rotor in time [12].

When selecting numerical techniques, their numerical stability is of primary importance. Solution of a system of equations of generator rotor movement, i.e. identification of generator rotor angular displacement in time, is possible by the method of successive intervals. However, this method has a material weaknesses that is in accumulating a considerable error at comparatively large duration of intervals, and therefore its use is usually limited by the study of the first cycle of rotor swinging [13], which is unacceptable when studying a generator operation mode with the purpose of identifying chaotic modes or its operation for long time of simulation.

Simulation of large systems with nonlinear difference equations is possible on computers with parallel architecture with the use of respective numerical techniques based on parallel computations. There are many publications related to it, e.g., [14, 15, 16, 17].

In this paper, with the use of results of work [14] and equation of generator movement working for SUP, and also using mathematical possibilities of NI LabVIEW [18] environment, a task was set - to design a virtual device (VD) and demonstrate its work for numerical study and simulating modes of generator operation close to the borders of its stability area with the purpose of identifying a possibility of chaotic oscillation of its rotor in the given operation mode on specific examples. 


\section{Numerical solution of generator nonlinear equation}

Equation of generator movement working on SUP and its electric power are recorded as follows [12]:

$$
\begin{gathered}
\frac{\tau_{J}}{\omega_{\text {ном }}} \frac{d^{2} \delta}{d t^{2}}=\frac{2 H}{\omega_{\text {ном }}} \frac{d \omega}{d t}=P_{\mathrm{T}}-P_{\text {эл }}, \\
P_{\text {эл }}=P_{m} \sin (\delta),
\end{gathered}
$$

where $T_{J}=2 \mathrm{H}$ - in seconds; $H$ - machine inertia constant; $\omega$ - angular frequency and its rated value $\omega_{H}-$ in radian/seconds; $d^{2} \delta / d t^{2}$ - angular acceleration in $\mathrm{rad} / \mathrm{s}^{2} ; P_{\mathrm{T}}, P_{\text {эл }}$ - power of primary engine and electromagnetic generator capacity in relative units respectively.

Equation of motion (1) in the initial mode recorded for a specific synchronous generator working on SUP via transformer and double-circuit power line, is with $P_{\mathrm{T}}=0,8, P_{m}=2,222$ as follows [12]:

$$
\frac{10}{377} \frac{d^{2} \delta}{d t^{2}}=(0,8-2,222 \sin \delta)
$$

Basic value of angle $\delta_{0}=21,09^{\circ}=0,367 \mathrm{rad}$. Rotor initial acceleration:

$$
\frac{d^{2} \delta}{d t^{2}}=37,7[0,8-(2,222 \sin 0,367)]=0,102 \mathrm{rad} / \mathrm{s}^{2}
$$

Equation (3) was formed from [1] for system (fig.1,a) on the basis of the scheme of its replacement (fig.1,б) and the following baseline data:
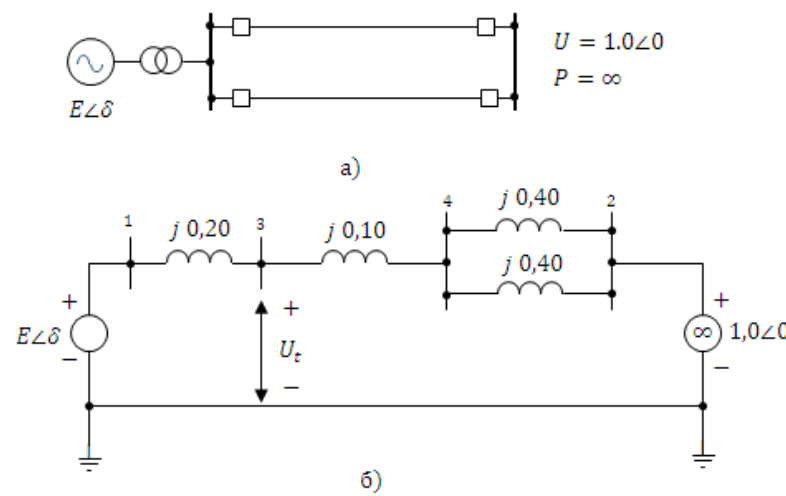

Fig.1. Scheme of the system a) and scheme of its replacement in the basic mode б)

voltage on system buses $U=1$; generator reactive transient resistance along longitudinal axis -0.2 ; transformer reactive impedance- 0.1 ; reactive impedance of each of two power transmission lines -0.4 , all values are related to baseline value of generator rated apparent power. In the basic mode, the machine gave active power of 0.8 , voltage on terminals 1.05. Machine inertia constant $H=5 M J /(M V * A)=5 s$. Active resistance is neglected.

Equations $(3,4)$ will be used for demonstrating dynamic behavior of generator close to borders of its stability area with the use of LabVIEW environment.

Let's replace state variables $\delta$ and $d \delta / d t=\omega(t)$ with $x_{1}$ and $x_{2}$ respectively, motion equation (1) with two first-order difference equations:

$$
\begin{aligned}
& \dot{x}_{1}=x_{2}-\omega_{\text {ном }}, \\
& \dot{x}_{2}=-a \sin x_{1}+b
\end{aligned},
$$

where $a=\left(P_{m} \omega_{\text {ном }}\right) / T_{J}=\left(P_{m} \omega_{\text {ном }}\right) / 2 H$; $b=\left(P_{\mathrm{T}} \omega_{\text {ном }}\right) / T_{J}=\left(P_{\mathrm{T}} \omega_{\text {ном }}\right) / 2 H ; \omega_{\text {ном }}=$ const .

Let's note that while preparing a block diagram for the task to be solved in LabVIEW environment, it is necessary to remember that $\omega(t)=d \delta / d t+\omega_{\text {ном }}$.

Use of LabVIEW standard functions for integrating a system of equations determines representation of the right part of difference equation (6) in the form of a set of text constants which makes it difficult to make changes into the numerical values of certain parameters that should be made when studying the impact of, for instance, change in electric power upon generator dynamic stability. Therefore, this paper uses equation solver given in paper [14].

Authors of the paper [14] called the numerically stable and quick operating solver for parallel method a second-order method with diagonal correction (hereinafter - DC). Algorithm of method in solution of system (5) is as follows:

$$
\left.\begin{array}{l}
x_{11}=x_{1}[n]+x_{2}[n] h ; \\
x_{21}=x_{2}[n]+\left(b-\sin x_{11}\right) h ; \\
x_{22}=x_{2}[n]+\left(b-\sin x_{1}[n]\right) h ; \\
x_{12}=x_{1}[n]+x_{22} h ; \\
x_{1}[n+1]=\left(x_{11}+x_{12}\right) / 2 ; \\
x_{2}[n+1]=\left(x_{21}+x_{22}\right) / 2,
\end{array}\right\}
$$

where $h$ - integration step value.

Realize algorithm (6) as a virtual device of LabVIEW simulation environment. Front panelinterface of the virtual device-is shown on Fig.2:

1- placed are elements for entering simulation parameters (duration of simulation and integration step in seconds, baseline value of rotor angle in radians, mechanical and electric power in relative units, angular frequency in $\mathrm{Hz}$ ); 2 - (XY Graph) graphical indicator for displaying of a phase pattern (along axis $\mathrm{X}$-change of rotor angle in degrees; along axis Y-rotor angular speed in degr./s); 3 - virtual oscillograph for displaying dependency of rotor angle on time; 4 - graphical indicator for displaying a range of input signal; 5 - these elements display values of frequency and amplitude of the found harmonic motion; 6 - button, if pressed displayed are options of displayed output signals, such as baseline signal; baseline signal harmonic component; baseline signal residual component. 


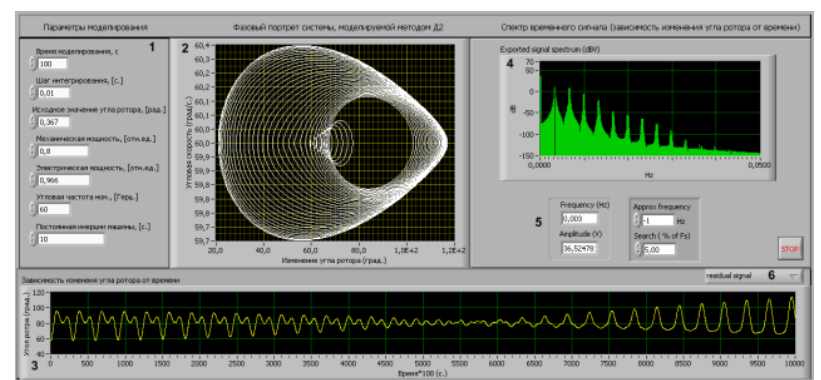

Fig.2. Front panel of VD for studying generator dynamic stability and its chaotic behavior

From among many functions of LabVIEW that were used in the designing VD, let's note only the function of measuring oscillograms' parameters "Extract Single Tone Information" ("Extract information about a single harmonic motion").

Thanks to Extract Single Tone Information function, a researcher can distinguish from rotor basic motion its components with VD, i.e. a single harmonic motion with the highest amplitude and residual signal, or search for certain range of frequencies and returns frequency, amplitude and phase of such oscillation.

Let's consider the change of rotor angle in time $\delta(t)$ and phase pattern of a discrete system, (6) on the example of equation of machine motion (2), simulated with VD numerical method, with the following baseline parameters of simulation: baseline value of rotor angle $\delta_{0}=21,09^{\circ}=0,367 \mathrm{rad}$ and its initial acceleration $0,102 \mathrm{rad} / \mathrm{s}^{2}$ (4); mechanical capacity $P_{\mathrm{T}}=0,8$; electric power $P_{m}=2,222$; rated angular frequency $\omega_{\text {ном }}=377 \mathrm{rad} / \mathrm{s} ;$ machine constant inertia $H=5 \mathrm{~s}$; computations were made with DC method with time integration step of 0.0001 .

The presented virtual device allows a researcher to simulate the work of generator and analyze the impact of all parameters of simulation upon its operating mode.

Let's set the task of identifying the degree of impact of $P_{m}$ values parameters in generator motion equation (2) upon its operating mode. To solve the task, use the designed VD for performing a simulation exercise, varying the value of parameter $P_{m}$ with unchanged values of others.

\section{Results of simulation exercise}

Results of simulation exercise are given in tab. $(1,2,3$, $4,5)$. First line of each table1-5 includes a graph of rotor angle change $\delta(t)$ in time, second and third lines - its components, i.e. single harmonic oscillations with the highest amplitude and residual components. They were obtained, as was stated above, thanks to using Extract Single Tone Information function ("Extract information about a single harmonic motion") in the virtual device.

Right part of the table contains phase patterns and dependency ranges $\delta(t)$. Phase patterns represent phase trajectories. Coordinates of each trajectory point show values of angle $\delta$ or rotor in degrees (axis of X) and its angular speed in degr/s ( $\mathrm{Y}$ axis) at the given moment of time $t$.

Table 1 Graphic expression of generator operating mode with $P_{\mathrm{T}}=0,8 ; P_{m}=2,222$

\begin{tabular}{|c|c|c|}
\hline & $\begin{array}{c}\text { Law of rotor } \\
\text { angle change in time } \\
(1) \text { and its } \\
\text { components }(2,3)\end{array}$ & $\begin{array}{c}\text { Phase } \\
\text { patterns and } \\
\text { range }\end{array}$ \\
\hline $\begin{array}{c}1 \\
\delta(t)=\delta_{1}(t)+\delta_{2}(t)\end{array}$ & \\
\hline 2 & \\
\hline$\delta_{1}(t)$ & \\
\hline$\delta_{2}(t)$ & \\
\hline
\end{tabular}

Table 1 presents the results of simulation exercise under rated mode of generator operation.

Graphic expression of generator operating mode with $P_{\mathrm{T}}=0,8 ; P_{m}=1,000$ is shown in tabl.2. In this mode, phase pattern is distorted, dependency $\delta(t)$ is other than sine curve, accordingly, additional harmonics appear and oscillation range is discrete.

Table 2 Graphic representation of generator operating mode with $P_{\mathrm{T}}=0,8 ; P_{m}=1,000$

\begin{tabular}{|c|c|c|}
\hline & $\begin{array}{c}\text { Law of rotor angle } \\
\text { change in time }(1) \text { and its } \\
\text { components }(2,3)\end{array}$ & $\begin{array}{c}\text { Phase pattern } \\
\text { and range }\end{array}$ \\
\hline \begin{tabular}{c}
$\delta(t)=\delta_{1}(t)+\delta_{2}(t)$ \\
\hline
\end{tabular} & \\
\hline$\delta_{1}(t)$ & & \\
\hline$\delta_{2}(t)$ & & \\
\hline
\end{tabular}

Tables 3, 4 show results of simulation exercise while simulating dynamic behavior of a generator close to borders of its stability area. The range of oscillations is non-discrete, but is becoming a continuous and wideband one.

In this mode, chaotic oscillations in the phase area of the trajectory develop continuously in time without 
Table 3 Graphic representation of generator operating mode with $P_{\mathrm{T}}=0,8 ; P_{m}=0,96337$

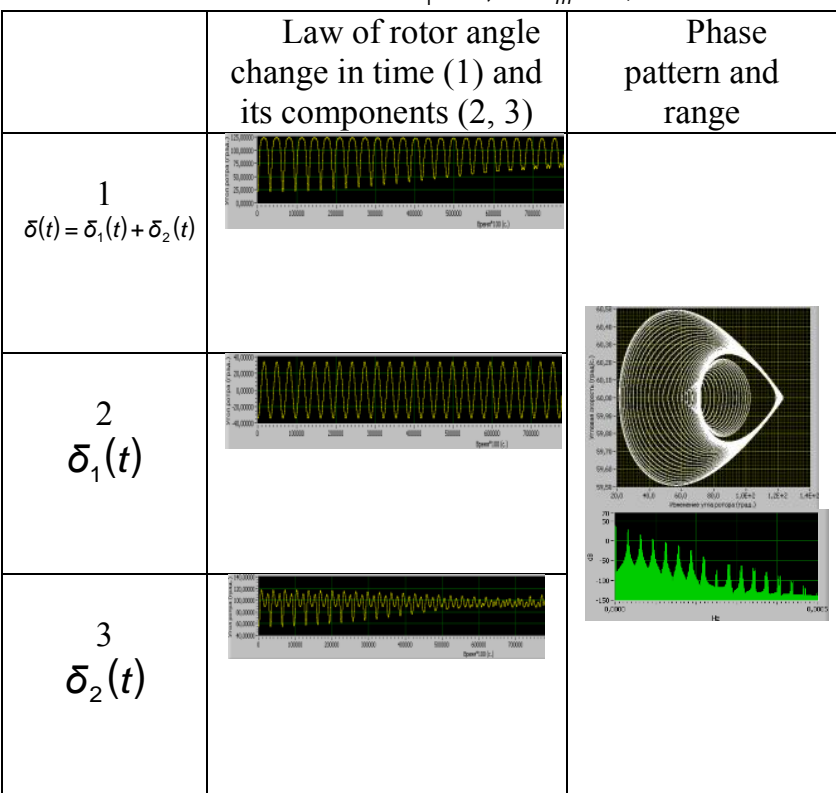

self-crossing and gradually fill some of its area so that any indefinitely small area of phase pattern is crossed by infinitude of trajectory segments. This is what creates a random situation - chaos - in each area [6].

Range of frequencies changes from discrete into continuous, which proves the presence of large number of frequencies some of which may match with own frequency of generator shaft mechanical oscillation. Such matching in its turn may cause resonance oscillation that is extremely undesirable for the generator.

Table 4 Graphic representation of generator operating mode with $P_{\mathrm{T}}=0,8 ; P_{m}=0,9633518548$

\begin{tabular}{|c|c|c|}
\hline & $\begin{array}{c}\text { Law of rotor angle } \\
\text { change in time (1) } \\
\text { and its components } \\
(2,3)\end{array}$ & $\begin{array}{c}\text { Phase pattern } \\
\text { and range }\end{array}$ \\
\hline $\begin{array}{c}|c| \\
\delta\end{array}(t)=\delta_{1}(t)+\delta_{2}(t)$ & & \\
\hline 2 & & \\
\hline$\delta_{1}(t)$ & & \\
\hline 3 & & \\
\hline$\delta_{2}(t)$ & & \\
\hline
\end{tabular}

Table 5 Graphic representation of generator operating mode with $P_{\mathrm{T}}=0,8 ; P_{m}=0,9633518547$

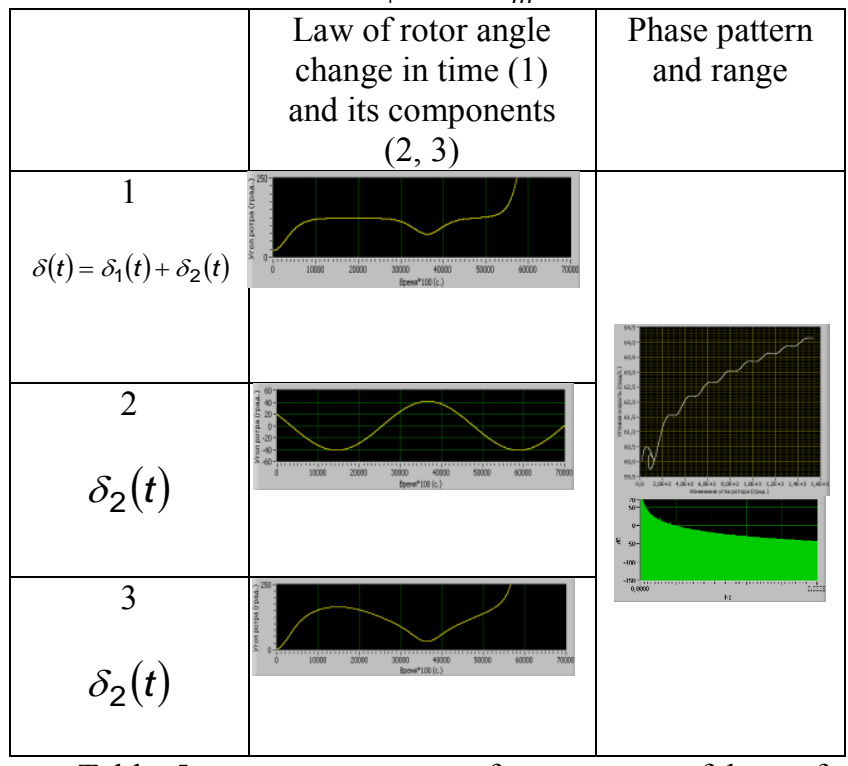

Table 5 represents a case of occurrence of loss of generator operation stability.

\section{Conclusion}

The article presents the results of a study providing the picture of alteration of the dynamic behavior of simulation (3) of generator with change in parameter $P_{m}$

. When the parameter was decreased from baseline value $P_{m}=2,222$ and when approaching value $P_{\mathrm{T}}=0,8$, complex dynamics phenomena are observed which are related to occurrence of various attractors (tab.1-5). In the phase area (tab.1-2) stable mode is matched by the system state of equilibrium. Asynchronous mode (tab.3) is matched by a multitude of stable multiturn rotary cycles, where phase point jumps from one cycle to another. A chaotic attractor (tab.4) is matched by a mode when $\delta(t)$ is changed stochastically and the range comprises multitude of narrow lines corresponding to oscillations themselves, and a wide base corresponding to "random" oscillations. In this mode, it is possible for the highest harmonics of current and voltage to appear. It was found out that loss of mode stability is observed in the system (tab. 5) when $P_{m}=0,963351857$.

So, the obtained results are of interest as concerns extending the horizon of knowledge about generator possible behavior. Especially important appears the found existence of areas of parameters values, when the system demonstrates chaotic attractors of various types.

Chaotic mode makes especially difficult the operation of synchronous generators, since chaotic modes have wideband range of frequencies and may induce harmonics of current and voltage that are dangerous for functioning of synchronous generators. 


\section{References}

1. L.O. Chua, Computerized analysis of electronic circuits: algorithms and computational method / L.O.Chua - M.: Energiya, p. 640 (1980).

2. H.D.Chiang, Chaos in simple power system / H.D. Chiang. IEEE Trans. Power Syst., Vol. 8(4), pp. 1407-1417 (1993).

3. S.Wiggins, Introduction to Applied Nonlinear Dynamical Systems and Chaos. Springer-Verlag, Telos, (1997).

4. H.G., Schuster, W. Just Deterministic Chaos: An Introduction, 4th edition. WILEY-VCH Verlag GmbH \& Co. KGaA, Weinheim, (2005).

5. J.C.Sprott, Elegant Chaos: Algebraically Simple Chaotic Flows. World Scientific, Singapore (2010).

6. V.K. Fedorov, Deterministic chaos in nonlinear electric circuits and systems. - Omsk: OmSTU, p. 130 (2006).

7. S.V.Sidorov, Structure of solutions and dynamic chaos in nonlinear difference equations // Vestnik RUDN. Series Math.. Inform.. Phys..- pp. 4563, 2 (2013).

8. V.K. Fedorov, Introduction to the theory of chaotic modes of nonlinear electric circuits and systems / V.K. Fedorov. - Omsk: OmPU, p. 144, (1992).

9. V.K. Fedorov, Peculiarities of energy dissipation in nonlinear electric circuits / V.K. Fedorov, P.V. Rysev and E.Yu. Sveshnikova // Omsk. Nauch. Vest. 1(30). pp.131-135 (2005).

10. D.V.Rysev, Stability of power system turbinegenerator - load at electromechanical resonance occurrence / D.V.Rysev and V.K. Fedorov // Omsk. Nauch. Vest. 3(103). pp. 194-198 (2011).

11. D.Zh. Baibagysova and K.Satarkulov, Virtual device for examining spectral composition of voltage in overhead line in chaotic mode of operation of power grid and computation of additional loss // KR HEIs News. 6. p 20-29 (2016).
12. P. Anderson and A. Fuad A., Operation of energy systems and stability / Translation from Eng. under editorship of Ya.N.Luginskiy. M.: Energiya, p.568, illus. (1980)

13. I.M. Markovich, Modes of energy systems. M.; L.: Gosenergoizdat, p. 271 (1957)

14. D.N. Butusov, V.Yu. Ostrovskiy and A.V. Krasilnikov, Simulation of nonlinear dynamic systems using parallel numerical techniques of integration // Basic research (Engineering sciences). №12, pp.18731877 (2014).

15. D.N. Butusov, A.I. Karimov, T.I Karimov and G.K. Dolgushin, Family of hardware-based methods of numerical integration // Modern issues of science and education. 4 [Electronic resource] (2014)

URL: http://www.scienceeducation.ru/ru/article/view?id=13480 (referral date: 27.05.2018).

16. K.G. Zhukov. Algorithm of embedding parallel calculations by formulas of Runge-Kutta numerical integration // Scientific and engineering bulletin of Saint-Petersburg State Polytechnic University. Informatics. Telecommunications. Managements. SPSPU, V. 6.2, 138. pp. 143-149 (2011)

17. L.P. Feldman, Parallel algorithms of simulation of dynamic systems described by simple difference equations// Electronic simulation. V. 26, 1. pp. 19-30 (2004).

18. A.O. Sevostyanov and V.V. Karataev. Using LabVIEW environment in studies of transient phenomena in nonlinear circuits with piecewise linear characteristics [Electronic resource]. URL: http://do.gendocs.ru/docs/239552.html

(date of referral: 07.08.2018) 